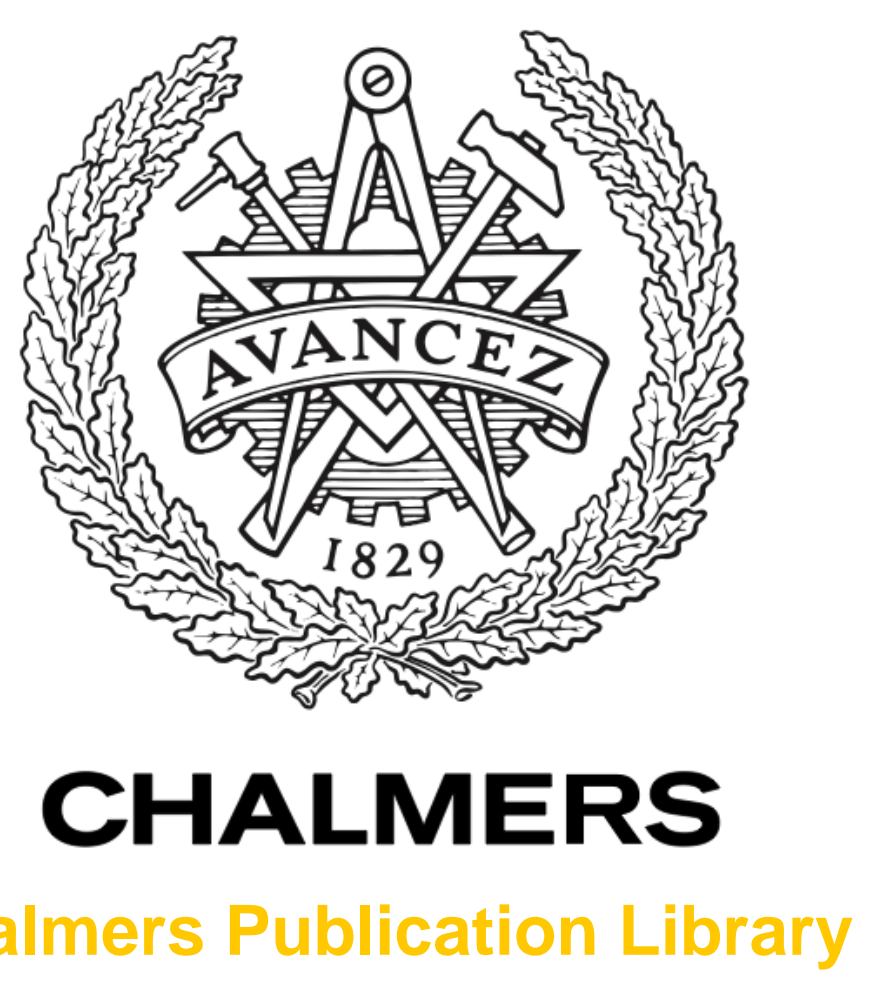

Ground-based GNSS-R solutions by means of software defined radio

This document has been downloaded from Chalmers Publication Library (CPL). It is the author's version of a work that was accepted for publication in:

Proceedings of the 2016 IEEE International Geoscience and Remote Sensing Symposium (IGARSS 2016)

Citation for the published paper:

Hobiger, T. ; Haas, R. ; Strandberg, J. (2016) "Ground-based GNSS-R solutions by means of software defined radio". Proceedings of the 2016 IEEE International Geoscience and Remote Sensing Symposium (IGARSS 2016), vol. NA(NA), pp. NA.

Downloaded from: http://publications.lib.chalmers.se/publication/242071

Notice: Changes introduced as a result of publishing processes such as copy-editing and formatting may not be reflected in this document. For a definitive version of this work, please refer to the published source. Please note that access to the published version might require a subscription. 


\title{
GROUND-BASED GNSS-R SOLUTIONS BY MEANS OF SOFTWARE DEFINED RADIO
}

\author{
T. Hobiger, R. Haas \& J. Strandberg \\ Chalmers University of Technology \\ Department of Earth and Space Science \\ SE-412 96, Gothenburg, Sweden.
}

\begin{abstract}
Usually ground-based GNSS-R installations are either existing geodetic GNSS stations or they are built with dedicated components that enable the deduction and monitoring of physical and geometrical properties of the reflecting area around that particular site. In both cases, hardware components usually enable real-time operation of such instruments. However, as software-defined radio (SDR) technology has advanced in the recent years it is now possible to carry out signal processing in real-time, which makes it an ideal candidate for the realization of a flexible GNSS-R system. It is shown how SDR can help to realize GNSS-R solutions for sea-level monitoring at the Onsala Space Observatory, Sweden. Moreover, such SDR solutions can be mounted on an unmanned aerial vehicle (UAV) in order to collect data from higher altitudes and even provide Delay-Doppler information for extended GNSS-R studies.
\end{abstract}

Index Terms - GNSS-R, software defined radio, reflectometry, GPU, UAV

\section{INTRODUCTION}

As for ground-based GNSS-R one can distinguish between two different concepts. First, there are systems which receive direct and reflected signals via one single antenna and require the extraction of reflector heights from the interpretation of signal-to-noise ratio (SNR) data (see e.g. $[1,2])$. In these cases usually commercial geodetic GNSS equipment is used to obtain information about reflector heights. As a second method of retrieving ground-based sea-level measurements one can utilize two antennas, one up-looking antenna for receiving the direct signal from the satellites and one downlooking antenna which is dedicated to receiving reflected signals from the water surface (cf. [3, 4]).

Also for this method commercial GNSS equipment is used, however sea-level heights are obtained from analysing carrierphase observations of the direct- and reflected signals. In general, it can be stated that single (up-looking) antenna studies are usually carried out when existing ground-based GNSS infrastructure has already been deployed and its data can be easily accessed. Concepts with two antennas are usually developed when reflected signals need to be processed by dedicated algorithms and/or when the scattered power is low and only high-gain downward looking antennas can help to detect ground reflections.

\section{GNSS-R INSTALLATIONS AT THE ONSALA SPACE OBSERVATORY}

The Onsala Space Observatory (OSO), located on the Swedish west coast, about $40 \mathrm{~km}$ south of Gothenburg, operates a GNSS-R system since several years. Regularly the local sealevel at the site is monitored and experiments are conducted that aim to improve the accuracy and precision of GNSS-R. Figure 1 depicts the location of the GNSS-R antennas as well as the recently build tide gauge that is part of the Swedish network of tide gauges operated by the Swedish Meteorological and Hydrological Institute (SMHI). Measurements from the SMHI tide gauge can be utilized in order to evaluate how well the GNSS-R solutions agree with this reference.

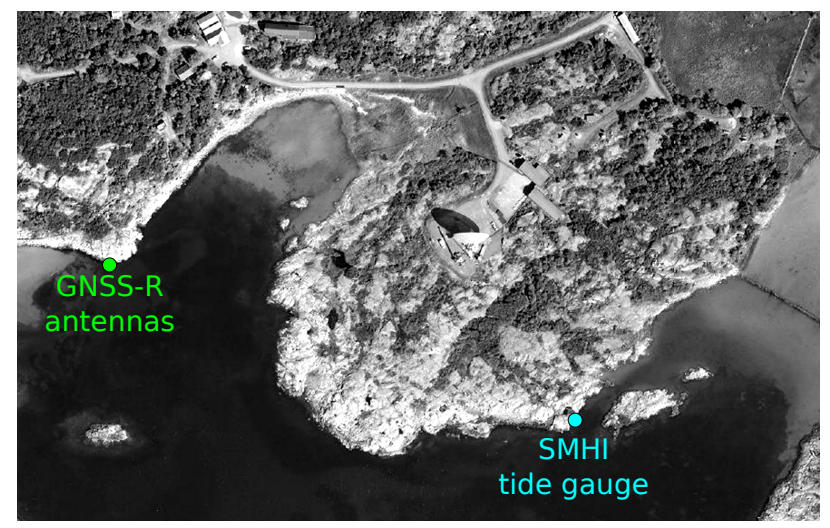

Fig. 1. Aerial image of the coastal area at the Onsala Space Observatory. The location of the GNSS-R system in the western part is marked with a green bullet and the location of the newly built SMHI tide gauge is marked in cyan. 


\section{GNSS-R BY MEANS OF SOFTWARE-DEFINED RADIO}

Software-defined radio (SDR) is a very powerful and flexible concept for prototyping and quick realization of projects without the need of application-specific integrated circuit (ASIC) boards. Thus, SDR is a very appealing solution which helps to implement a novel GNSS-R concept with much lower prototyping and development cost. As signal processing can be carried out on a PC, such a GNSS-R solution can be built with off-the-shelf components and adapted in a very flexible way before or during any development phase. As discussed in the following, SDR has been also chose for the development of new GNSS-R solutions at the Chalmers University and tested at the Onsala Space Observatory.

\subsection{GLONASS-R}

Correlation of direct and reflected signals is not possible without certain hardware changes. However, in the case of Russian Global Navigation Satellite System (GLONASS) which makes use of the frequency division multiple access (FDMA) encoding scheme of $\mathrm{cm}$-level precision from ground-based GNSS-R installations can be obtained [5]. However, the need for complex and expensive RF front-ends, down-conversion stages and A/D converters made the so-called GLONASS-R system rather unattractive for being duplicated at other sites. This problem is thought to be overcome by replacing those inflexible components with off-the-shelf software-defined radio equipment. This does not only lead to a drastic price reduction but also increases the flexibility of the GLONASS-R concept. Recent results from such a prototype system are shown in figure 2 and summarized in more detail in [6].

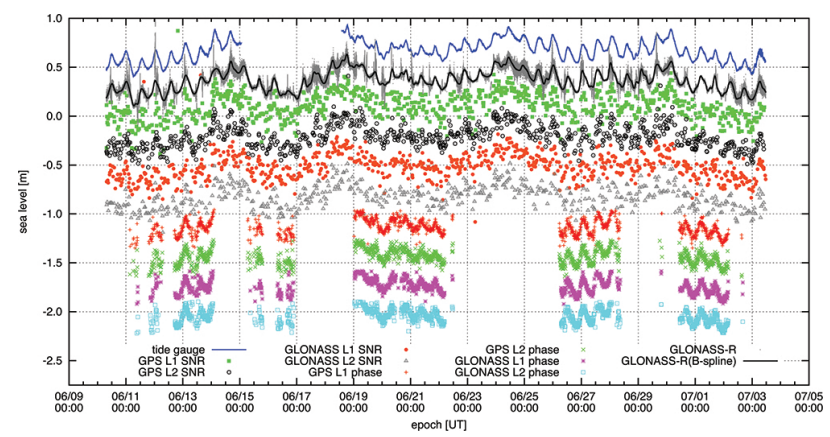

Fig. 2. Tide gauge measurements from 2015 are plotted together with GLONASS-R results and data obtained from the geodetic GNSS receivers. The latter include SNR and single difference solutions for GPS and GLONASS on both L1 and L2 frequencies. Mean values w.r.t. the tide gauge series have been removed, and to improve the readability of the figure the time series were shifted with offsets of $30 \mathrm{~cm}$.

\subsection{Miniaturization}

SDR based systems face one major restriction. Although SDR solutions are very flexible, a commodity PC is usually necessary to carry out most of the signal processing steps. Thus, the total size of GNSS-R solution depends on how much space sampler units and the PC occupy. A reduction in the size of the PC comes along with less powerful computing capacity, which would restrict the capabilities for real-time signal processing. In order to avoid such limitations one could off-load heavy signal processing stages to the FPGA. This would normally imply more complicated and longer development processes and counteract the idea of software defined radio. The pocket sized USRP E310 series from Ettus seems to fill this niche, as it can act as a stand-alone software defined radio. Moreover, RF Network on Chip (RFNoc) technology allows to move signal processing steps on the FPGA while still keeping all the advantages of SDR. Therefore, it is possible to reduce the requirements for the $\mathrm{PC}$ and even replace desktop size computer hardware with off-the shelf open hardware, embedded computers that are not bigger than the E310 itself.

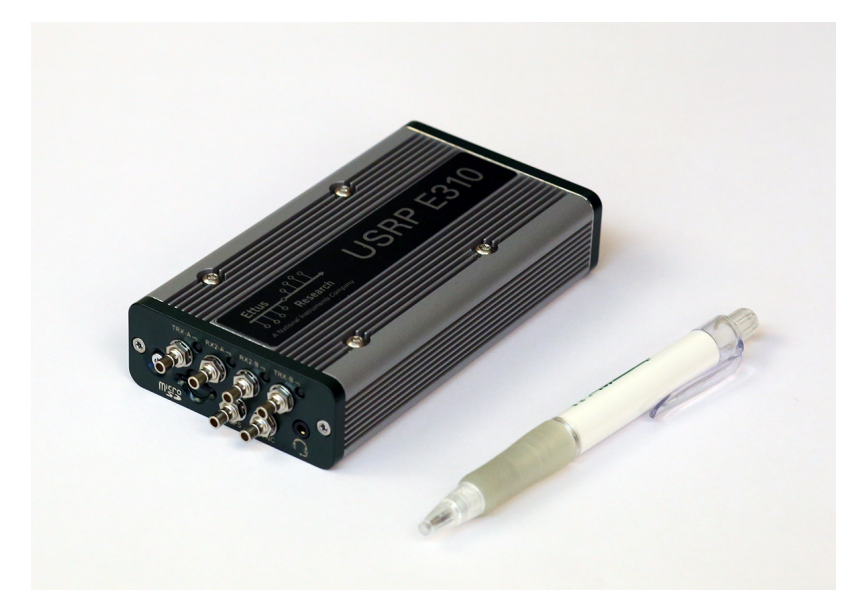

Fig. 3. Ettus' USRP E310 is a potential solution that could miniaturize our GNSS-R system. It provides direct-sampling in two channels and covers any of the complete range of GNSS frequencies. It's size is not larger than a modern mobile phone and it has a typical power consumption of 2-6 watts.

\section{OUTLOOK}

Given it's simplicity and the miniaturization steps described before, the SDR based GNSS-R solution could also be of use for air- or space-borne interferometric instruments. For such applications it would be necessary that the Doppler shift and spread of the reflected signal are handled properly and aircraft altitude is tracked and compensated with sufficient accuracy. This would then allow a straightforward determination of so- 
called delay-doppler maps which can be used for a variety of geophysical applications. A budget for the purchase of an unmanned aerial vehicle (UAV) has already been secured and first flight tests with a prototype system are expected until summer 2016.

\section{ACKNOWLEDGEMENT}

This research has been conducted with support from "Stiftelsen Lars Hiertas Minne" (FO2014-0663) and "Adlerbertska forskningsstiftelsen". The commercial GNSS-equipment (receivers, antennas) were purchased via the Leica Geosystems ATHENA program. The authors also thank teams from the workshop and the electronics laboratory at the Onsala Space Observatory for preparation of the infrastructure at the test site. We gratefully acknowledge the support of NVIDIA Corporation with the donation of the Tesla K40 used for this research.

\section{REFERENCES}

[1] K.M. Larson, R.D. Ray, F.D. Nievinski, and J.T. Freymueller, "The Accidental Tide Gauge: A GPS Reflection Case Study From Kachemak Bay, Alaska," Geoscience and Remote Sensing Letters, IEEE, vol. 10(5), pp. 12001204, 2013.

[2] J.S. Löfgren, R. Haas, and H.G. Scherneck, "Sea level time series and ocean tide analysis from multipath signals at five GPS sites in different parts of the world," Journal of Geodynamics, vol. 80, pp. 66-80, 2014.

[3] J.S. Löfgren, R. Haas, H.G. Scherneck, and M.S. Bos, "Three months of local sea level derived from reflected GNSS signals," Radio Science, vol. 46, pp. RS0C05, 2011.

[4] J.S. Löfgren and R. Haas, "Sea level measurements using multi-frequency GPS and GLONASS observations," EURASIP Journal on Advances in Signal Processing, vol. $1,2014$.

[5] T. Hobiger, R. Haas, and J.S. Löfgren, "GLONASS-R: GNSS reflectometry with a Frequency Division Multiple Access-based satellite navigation system," Radio Science, vol. 49, pp. 271-282, 2014.

[6] T. Hobiger, R. Haas, and J.S. Löfgren, "Software defined radio direct correlation GNSS reflectometry by means of GLONASS," IEEE Journal of Selected Topics in Applied Earth Observations and Remote Sensing, submitted. 